\title{
Spectroscopic Measurements in the Shock Relaxation Region of a Hypervelocity Mach Reflection
}

\author{
M. Sharma* J. M. Austin† N. G. Glumacªnd L. Massa ${ }^{\S}$
}

\begin{abstract}
We examine the spatial temperature profile in the non-equilibrium relaxation region behind a stationary shock wave. The normal shock wave is established through a Mach reflection configuration from an opposing wedge arrangement for a hypervelocity air Mach 7.42 freestream. Schlieren images confirm that the shock configuration is steady and the location is repeatable. Emission spectroscopy is used to identify dissociated species and to obtain vibrational temperature measurements using the $\mathrm{NO}$ and $\mathrm{OH} \mathrm{A}-\mathrm{X}$ band sequences. Temperature measurements are presented at selected locations behind the normal shock. LIFBASE is used as the simulation spectrum software for $\mathrm{OH}$ temperature-fitting, however the need to access higher vibrational and rotational levels for NO leads to the use of an in-house developed algorithm. For NO, results demonstrate the contribution of higher vibrational and rotational levels to the spectra at the conditions of this study. Very good agreement is achieved between the experimentally measured NO vibrational temperatures and calculations performed using a state-resolved, one-dimensional forced harmonic oscillator thermochemical model.
\end{abstract}

\section{Introduction}

At hypersonic conditions encountered during atmospheric planetary entry, molecular real gas processes become significant. Thermal transitions and chemical reactions are known to affect flight aerodynamics, for example the bow shock standoff distance in front of a blunt body is increased by chemical dissociation. ${ }^{1}$ Behind a strong shock, high temperatures and large radiative heating rates occur. The extent and profile of the post-shock relaxation region must be predicted to infer heat flux to the vehicle body. The complexity of a thermochemically active, hypersonic flow tests the current limits of computational modeling. ${ }^{2}$ Experimental data, particularly obtained using non-intrusive techniques, are therefore valuable, but conditions are typically challenging for measurement diagnostics.

In this study, the non-equilibrium relaxation region behind a stationary normal shock is investigated using spectroscopic radiation measurements. The choice of a normal shock eliminates flow field complexities associated with a curved bow shock, such as mixed subsonic-supersonic regions, entropy gradients due to flow curvature and three-dimensionality. With well-defined inflow conditions, this canonical flowfield provides a convenient testbed to evaluate thermochemical models and to demonstrate the applicability of a non-instrusive diagnostic technique for hypervelocity flows. The normal shock is created via a Mach reflection using an opposing wedge arrangement for a nominal Mach 7.4 air run condition using the Hypervelocity Expansion Tube (HET) facility at the University of Illinois at Urbana-Champaign. An impulse-type facility, the expansion tube is capable of accessing high-enthalpy conditions. Previous experimental studies have confirmed the presence of real gas effects behind the normal shock. ${ }^{3}$ In the present work, the nitric oxide A-X band (also referred to as the gamma, or $\gamma$ band) transitions are used to measure vibrational temperatures

\footnotetext{
* Graduate Student, Department of Aerospace Engineering, University of Illinois, Member AIAA

${ }^{\dagger}$ Assistant Professor, Department of Aerospace Engineering, University of Illinois, Senior Member AIAA

${ }_{\ddagger}^{\ddagger}$ Professor, Department of Mechanical Science and Engineering, University of Illinois, Senior Member AIAA

$\S$ Assistant Professor, Department of Mechanical and Aerospace Engineering, University of Texas, Senior Member AIAA
} 
within the relaxation region for comparison with calculations using a state-resolved thermochemical model.

In a partially dissociated and vibrationally excited freestream, Nerem et al. have measured relaxation times of dissociated air behind a normal shock for shock speeds between 4.9 to $6.8 \mathrm{~km} / \mathrm{s}$ by examining the radiative emission time-history. ${ }^{4}$ Vibrational temperatures and relaxation times behind normal shock waves, with shocked temperatures between 1400-3200K, were measured by Russo for CO test gas mixtures. ${ }^{5}$ Timeresolved vibrational temperature measurements of $\mathrm{CO}$ were made using the emission band-reversal method and the line-reversal method for seeded sodium by assuming equilibrium between $\mathrm{CO}$ vibrational and sodium electronic temperatures. In the Mach range of 3.5-5.7, Moser and Hindelang measured nitric oxide vibrational relaxation times behind incident normal shock waves. ${ }^{6,7}$ Moser and Hindelang employed infra-red diode laser absorption spectroscopy tuned to specific ro-vibrational transitions for a NO-Ar gas mixture. At shock velocities of $6.2 \mathrm{~km} / \mathrm{s}$, Sharma and Gillespie conducted rotational and vibrational temperature measurements behind a normal shock, ${ }^{8}$ which sought to advance previous non-equilibrium relaxation region measurements by Allen et al. ${ }^{9-11}$ In each of these cases, nitrogen was the test gas. Sharma and Gillespie completed their measurements using photomultiplier tube radiometers and the second positive system of $\mathrm{N}_{2}$. Using the Marseille free-piston shock tube and a shock velocity of $5.8 \mathrm{~km} / \mathrm{s}$, Labracherie et al. studied the non-equilibrium region downstream of a normal shock wave. ${ }^{12}$ Labracherie et al. presented the temporal evolution of vibrational and rotational temperatures and did not specifically investigate spatial temperature gradients. Temperature measurements were made for a methane-nitrogen-argon mixture using the CN B-X electronic transition. Using the spontaneous emission of the $\delta v=0$ band of $\mathrm{CN}$, Ramjuan et al. spatially measured the relaxation region behind a normal shock with a velocity of $5.6 \mathrm{~km} / \mathrm{s} .{ }^{13}$ Recently, Rond et al. used the $\mathrm{CN}$ violet system to show time-resolved post-shock intensity profiles and to measure post-shock vibrational temperatures using emission spectroscopy. ${ }^{14}$

These previous studies demonstate that emission spectroscopy is a viable technique for determining internal energy mode temperatures within a non-equilibrium flow field. $\mathrm{CO}_{2}$ flow fields were the focus of many of the experimental studies that utilized emission spectroscopy as the diagnostic technique and CN was the species of interest. In the current study, we examine air flows and NO as the emitter species. Experimental results from CUBRC (Calspan University of Buffalo Research Center), for shock velocities between 3-4 km/s, used emission spectroscopy to identify the NO A-X band as the dominant radiator behind a strong shocks for a $\mathrm{O}_{2}-\mathrm{N}_{2}$ gas mixture. ${ }^{15}$ A follow-up experimental study used the region behind the shock wave to determine the squared transition moment value of the NO gamma band and to obtain time-dependent NO gamma radiative signals. ${ }^{16}$ The calculation of internal energy mode temperatures using radiation signals from NO band systems via comparison with numerical spectra simulation has been successfully demonstrated on numerous occasions in air plasma experiments. In the NASA Ames $20 \mathrm{MW}$ arcjet wind tunnel, vibrational temperatures have been measured by using the emission spectra of the NO $\gamma(\mathrm{A}-\mathrm{X})$ and $\delta(\mathrm{C}-\mathrm{X})$ bands. ${ }^{17,18}$ In the same facility, rotational temperatures have also determined using the NO $\delta(0,4), \gamma(0,0), \gamma(0,1), \gamma(0,2)$ and $\gamma(0,3)$ bands. ${ }^{19}$ Using strong $\gamma$ and weaker $\beta$ (B-X), $\delta$ and $\epsilon(\mathrm{D}-\mathrm{X})$ band transitions, all internal energy mode temperatures were measured for plasma discharges in air. ${ }^{20}$

In the previous shock wave studies, measurements have been made behind propagating waves. In the present experiments, we instead establish a stationary shock using an opposing double wedge model geometry. The flowfield is first visulized using the schlieren technique. Shock angles are measured and since the free stream inflow conditions are known, the frozen post-shock state is well-characterized.

The shock-tube was almost exclusively the only type of impulse facility used in the previous studies discussed above. In reflected-shock tunnels, chemical freezing within the nozzle and freestream non-equilibrium effects can alter the test gas. ${ }^{21-23}$ An expansion tube is an alternate means of achieving high-enthalpy hypersonic conditions. Therefore, this work also serves as a testbed for demonstrating the applicability of emission spectroscopic techniques for temperature measurement within an expansion tube. Emission spectroscopy can provide useful data for facility characterization. At the TCM2 free-piston shock tunnel in France, emission spectra have been used to calculate useful test times and demonstrate test time homogeneity for different operating conditions. ${ }^{24,25}$ Test time determination and species identification in the JXI expansion tube facility using emission spectroscopy was performed by Ramjuan et al. ${ }^{26}$ 


\section{Experimental Setup}

\section{A. Hypervelocity Expansion Tube facility}

The HET is a $9.14 \mathrm{~m}$ long facility consisting of three sections (driver, driven, and accelerator) all with $150 \mathrm{~mm}$ inner bore diameter, initially separated by primary and secondary diaphragms. The initial gas pressure and composition can be varied to achieve nominal Mach number operating conditions between 3.0 to 8.5 and stagnation enthalpies of 4.5 to $8.0 \mathrm{MJ} / \mathrm{kg}$. In the present study, an air test gas, helium driver and accelerator gas run condition (labeled Air-5) with Mach number 7.42 is selected. Calculated test gas properties are shown in Table 1.

The facility is initially evacuated, then each section filled to the desired initial pressure. The primary diaphragm burst pressure (driver pressure) is measured using a Setra 206 pressure gauge. Driven and accelerator initial fill pressures are monitored using MKS Piezo+ A900-02 transducers. Vacuum pressures (below $2000 \mathrm{mTorr}$ ) in the accelerator section are measured using a MKS Baratron 626A transducer with 2 Torr fullscale, rated to $0.25 \%$ of the reading. This model was deliberately selected as the capacitance manometer pressure-measurement mechanism is invariant to the gas composition. Initial pressures in the accelerator section are typically on the order of 300 mTorr, and the influence of air-leakage into the acceleration section is mitigated by initially evacuating the section down to 200 mTorr, flushing with helium up to $12 \mathrm{kPa}$ and then performing the final evacuation down to the desired pressure. Within the facility, the acceleration section leak-rate was measured to be $0.3-0.4 \mathrm{mTorr} / \mathrm{min}$ and the subsequent partial pressure of air based upon these leak-rate measurements is low enough such that the effect is negligible based on perfect gas dynamic calculations. Characterization of the out-gassing phenomena for these set of experiments, yielded a maximum 7 mTorr increase after the final accelerator evacuation. Pressure data were recorded using a National Instruments data acquistion system, consisting of a PXI-1031 chassis, a BNC-2100 8 channel connector block and a 14-bit PXI-6133 $3 \mathrm{MS} / \mathrm{s}$ simultaneous sampling multi-function data acquisition module. A full description of the facility, measurement capabilities and facility operation can be found in Dufrene et al. ${ }^{27}$ Facilty operation has been previously characterized experimentally and numerically. ${ }^{27,28}$

\section{B. Imaging System and Spectroscopic Setup}

Mach reflection configurations were visulized using a schlieren system consisting of a Xenon nanopulser (10 ns duration) white light source and pco.1660 (Cooke Coorporation) CCD camera. The system is triggered via the transmitted shock arrival at a $1 \mathrm{MHz}$ response pitot pressure transducer (PCB 113A26) sting-mounted in the test section $31.75 \mathrm{~mm}$ below the model centerline. Schlieren images and pitot pressure traces from previous experiments ${ }^{27}$ combined with the computational results discussed below ensure a repeatable and steady Mach reflection during the test time.

For the spectroscopic measurements, the broadband emission light is collected and collimated at 90 degrees to the vertical axis plane of the test section with a $200 \mathrm{~mm}$ focal length, $50.8 \mathrm{~mm}$ diameter (f/4) UV coated lens, Figures $1 \mathrm{a}$ ) and b). The collimated light is then focused for an imaging spectrograph. Two spectrographs were used in this study: a f/2 CP140 Jobin Yvon spectrograph for measurements which will be referred to as "coarse", and a f/4 270M SPEX spectrograph for measurements which will be referred to as "fine". Spectral lines were visualized using a Princeton Instruments PI-MAX MG:512SB intensified CCD camera system and a ST-133 camera controller. For each run, the camera exposure was set to $80 \mu \mathrm{s}$ in order to capture as large a portion of the test gas as possible without accelerator or driver gas contamination. For the f/4 270M SPEX spectrograph measurements, a slit size of $43 \mu$ s was selected as a compromise between signal strength and resolution. At the focal plane of the spectrometer, the dispersion across the chip was $0.07 \mathrm{~nm} /$ pixel, corresponding to a wavelength range of $35.71 \mathrm{~nm}$. The measured resolution is 1.4 Angstrom. For the NO wavelength and intensity calibration, a hollow cathode Fe and a L6302 D2 Hamamatsu lamp with Oriel 6310 power supply are used respectively. For the $\mathrm{OH}$ wavelength and intensity calibration, an Oriel pencil style $6035 \mathrm{Hg}$-Ar lamp with a 6045 power supply and a 20W Newport 6319 tungsten lamp with a Oriel 68735 power supply are used respectively. Calibration sources were placed inside the test section 


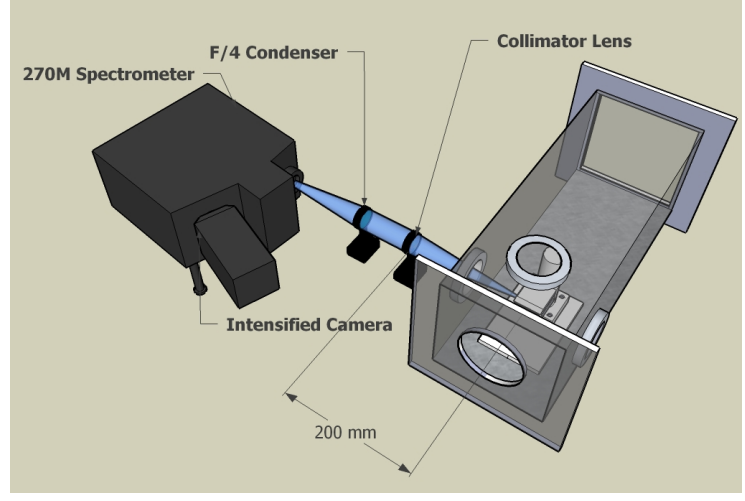

a)

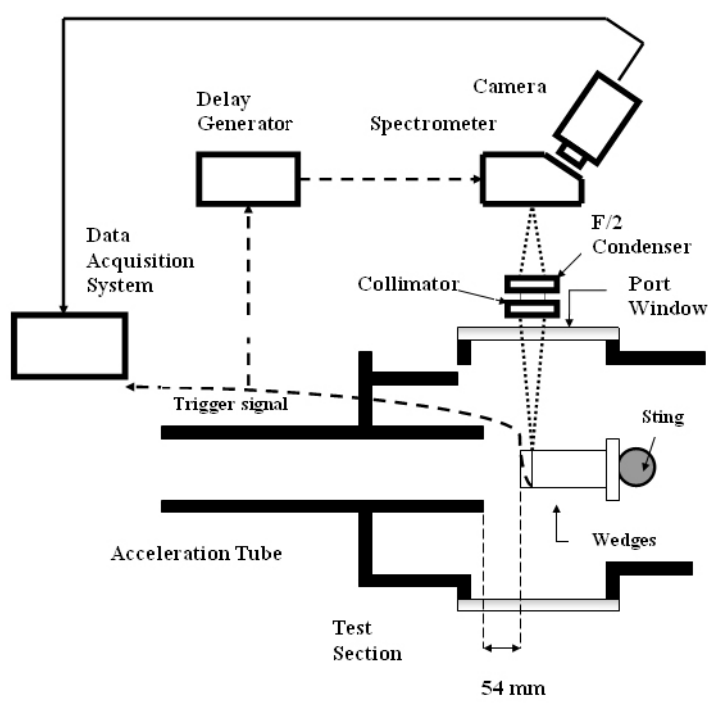

b)

Figure 1. a) Three dimensional schematic of imaging spectroscopy collection system. b)Schematic of experimental data acquisition setup

and imaging points located with $1 \mathrm{~mm}$ spatial precision via the use of fine-scale translation stages. To assist with data-processing, background intensity and wavelength spectra were also obtained for each shot. For the coarse resolution results, for which no intensity calibration was performed, the $6035 \mathrm{Hg}$-Ar pencil lamp was the wavelength calibration source. Two different slit sizes of 25 and $50 \mu \mathrm{m}$ were used for which only the $50 \mu \mathrm{m}$ results are presented. As with the schlieren system, the spectrometer acquisition sequence was triggered using the transmitted shock arrival at the test section pitot probe which was located within the core flow, $31.75 \mathrm{~mm}$ below the tube centerline. ${ }^{27}$

\section{Numerical Modeling}

The experimental results are compared with a calculations using an existing thermochemical model, ${ }^{29}$ based on the semiclassical,forced harmonic oscillator model with free-rotating (FHO-FR) molecule paths by Adamovich and co-workers. ${ }^{30-32}$ The molecule-molecule rates of Adamovich ${ }^{32}$ are extended to higher collisional energies by a curve fit function obtained by minimizing the deviation from the numerical integration of the transition probability over all possible $\mathrm{O}_{2}, \mathrm{~N}_{2}$ and $\mathrm{NO}$ transfers. The three-dimensional collisional model of Adamovich and Rich $^{30}$ is used for the molecule-atom rates. State-resolved chemical reaction rates are taken from Bose and Candler. ${ }^{33,34}$ In this work, Boltzmann distributions are assumed for NO produced by the first Zeldovich reaction. However, non-Boltzmann distributions are modeled for NO produced by the second Zeldovich reaction. ${ }^{34}$ The thermochemical model was validated by comparision with experimentally obtained data for rate constants, ${ }^{35,36}$ vibrational relaxation times, ${ }^{37,38}$ dissociation rates ${ }^{39,40}$ and VV transfer probabilities. ${ }^{41}$ Using the master equation approach of Park, ${ }^{23}$ a total of 37,48 , and 40 vibrational levels for $\mathrm{O}_{2}, \mathrm{~N}_{2}$, and $\mathrm{NO}$ respectively are obtained in addition to monatomic $\mathrm{O}$, N, and Ar, resulting in a 128 species mixture. Initial inflow conditions for the calculations (static pressure, static temperature and test gas Mach number) are obtained from inviscid, perfect-gas calculations of expansion tube operation. 


\section{Results}

\section{A. Test Gas Freestream Condition}

Table 1 summarizes the test gas freestream condition with a comparision between experimentally measured values and inviscid, one-dimensional, perfect gas calculations. The experimental values presented are ensemble averaged across a total of 28 separate events, consisting of 20 experiments for NO spectra acquisition and 8 experiments for $\mathrm{OH}$ spectra acquisition. Across most of the test condition parameters the agreement between theoretical expectation and measurement is very good. The largest discrepancy is seen in the calculated and measured values of the transmitted shock velocity, $\mathrm{u}_{t}$. However, two-dimensional Navier-Stokes simulations of a very similar run condition (Air-1) have shown that the transmitted shock is considerably attentuated due to viscous effects through the acceleration section. ${ }^{28}$ Good agreement was obtained between the numerically predicted and the experimentally measured velocity. The previous run condition, Air-1, differs from Air-5 only by the primary diaphragm burst pressure. Perfect gas predictions indicate that the transmitted shock velocity differs by only $1 \%$ for the change in burst pressure. Therefore, $u_{t}$ is assumed to be invariant with burst pressure and the calculated $\mathrm{u}_{t}$ overprediction results from the inviscid assumption. The experimental test gas Mach number is calculated from the measured pitot pressure using the theoretical prediction of the static test gas pressure, $\mathrm{p}_{7}$. Very low signal-to-noise ratios prevent experimental measurement of $\mathrm{p}_{7}$ for the current run condition. Despite a lower than theoretically predicted test-time, a sufficient slug of test gas exists in which to make spectroscopic measurements.

\begin{tabular}{|l|l|l|l|l|l|l|l|l|l|}
\hline & $M_{7}$ & $\begin{array}{l}h_{o, 7} \\
(\mathrm{MJ} / \mathrm{kg})\end{array}$ & $\begin{array}{l}\text { Test } \\
\text { Time } \\
(\mu \mathrm{s})\end{array}$ & $\begin{array}{l}P_{7, p i t o t} \\
(\mathrm{kPa})\end{array}$ & $\begin{array}{l}P_{7} \\
(\mathrm{~Pa})\end{array}$ & $\begin{array}{l}u_{t} \\
(\mathrm{~m} / \mathrm{s})\end{array}$ & $\begin{array}{l}u_{7} \\
(\mathrm{~m} / \mathrm{s})\end{array}$ & $\begin{array}{l}u_{s} \\
(\mathrm{~m} / \mathrm{s})\end{array}$ & $T_{7}(\mathrm{~K})$ \\
\hline Experiment & 7.30 & - & 104 & $53.64 \pm 6.1$ & - & $4148 \pm 47$ & - & $2044 \pm 42$ & - \\
\hline Theoretical & 7.42 & 7.88 & 163 & 55.6 & 778 & 5228 & 3777 & 1995 & 644 \\
\hline
\end{tabular}

Table 1. Selected test gas freestream parameters. Theoretical calculations assuming one-dimensional, perfect gas dynamics are compared with experimental measurements.

\section{B. Asymmetric Mach Reflection}

A Mach reflection is created by an asymmetric wedge configuration as shown in the schlieren image of Figure 2 a), which displays the Mach stem bridging two triple points from which free shear layers emanate. 25 and 35 degree half-angle wedges are used with a tip-to-tip separation of $25.4 \mathrm{~mm}$. In order to avoid three-dimensional effects, inlet aspect ratios of 1.25 and wedge aspect ratios of 5 were used. ${ }^{42}$

The Mach reflection (MR) has been extensively studied throughout the literature as an example of a shock-shock interaction which occurs extensively in aerodynamic appplications. With significant thermochemical effects present in the current study, it is important to characterize the anticipated influence upon the Mach reflection configuration. Numerous previous studies have confirmed that the overall MR configuration is displaced forward and the Mach stem height is decreased in the presence of non-equilibrium effects. ${ }^{43-46}$ The Mach stem height reduction increases the experimental difficulty as the post-shock measurement area is also decreased. Numerical simulations of the current asymmetric wedge arrangement indicate that the flow field is significantly dependent upon the thermochemical model ${ }^{47}$ Frozen, finite-rate and equilibrium models were examined. The thermochemical model was seen to change both the location and height of the Mach stem. These results are consistent with the effects of thermochemistry previously observed in the literature. Finite-rate simulations provided the best agreement with experiment. However, the simulation predicted a slightly further recessed and longer Mach stem than experimentally observed from the schlieren imaging. The simulations also indicated that the Mach stem position is stationary $20 \mu$ s after the passing 


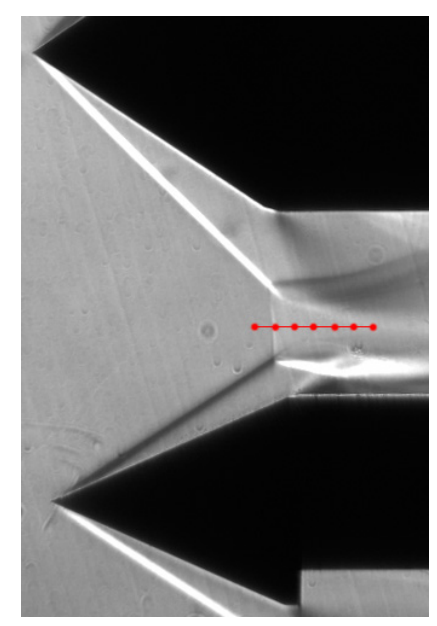

a)

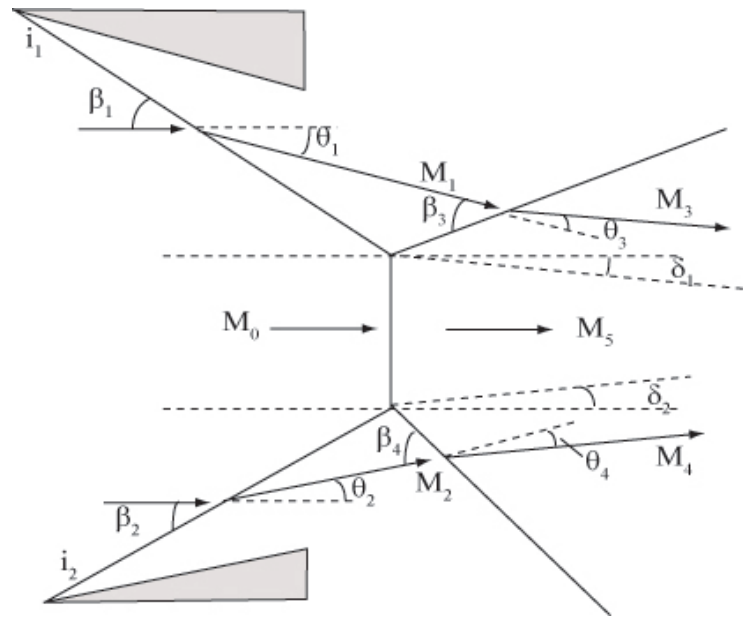

b)

Figure 2. a) Schlieren image of an asymmetric Mach reflection in Air-5 test condition, nominal freestream Mach number of 7.42. Emission spectroscopy measurement locations are indicated. b) Schematic of an asymmetric Mach reflection.

of the contact surface. For a test gas duration of approximately $120 \mu$ s this confirms that a steady Mach reflection exists and ensures, combined with a consistent triggering event, that the steady portion of the test gas is captured.

From schlieren images, the Mach stem is measured to be $3.9 \mathrm{~mm}$ high, almost perpendicular,located $12.7 \mathrm{~mm}$ downstream of the wedge tip plane and $2 \mathrm{~mm}$ below the tube axial centerline (which also corresponds to the axial centerline of the wedges). As Figure 2 a) shows, the uniform region behind the Mach stem which is not influenced by the converging shear layer slipstreams is very small (approximately $5 \mathrm{~mm}$ ). It is therefore imperative that the spatial location of the collection area is known very precisely with respect to the Mach stem. This involves determining the repeatability of the Mach stem location and potential shift from experiment-to-experiment. Results found the deviation on both height and location measurements to be $\pm 0.5 \mathrm{~mm}$. This experiment-to-experiment movement is deemed small enough with respect to uniform region length and the Mach stem height such that the calibration imaging point (acquired before the experiment) corresponds to the same location within the flowfield during the experiment. Measurements were made along the Mach stem centerline at -1, 0, 1, 2, 3, 4 and $5 \mathrm{~mm}$ downstream of the Mach stem, locations indicated by the symbols in Figure 2 a).

The MR configuration geometry has been verified in the via measurement of shock angles from the schlieren images. The results are presented in Table 2 and compared against perfect gas predictions. The subscripts refer to the Mach reflection schematic depicted in Figure $2 \mathrm{~b}$ ). The theoretical reflected-shock angles were determined from shock-polar calculations. Comparisons yield very good agreement between incident shock angles. The differences observed for the lower triple-point reflected-shock and slipstream angles are more pronounced, especially so for the slipstream angle. Whilst the static test gas temperature has not been directly measured, the agreement between test gas pitot pressure, test gas Mach number and incident shock angles suggests that freestream conditions are well-predicted by perfect gas theory.

\section{Species Identification}

A spectrum obtained $10 \mathrm{~mm}$ downstream of the Mach stem over the wavelength regime of 200-400 $\mathrm{nm}$ using the f/2 CP140 Jobin Yvon spectrograph is shown in Figure 3. Coarse resolution acquisition was undertaken as a means identifying potential temperature-measurement appropriate species. Emission from the $\mathrm{OH}$ radical 


\begin{tabular}{|l|l|l|}
\hline Shock Angles (degrees) & Theory & Exp. \\
\hline$\beta_{1}$ & 46.6 & $44.4 \pm 0.3$ \\
\hline$\beta_{2}$ & 33.0 & $32.5 \pm 0.3$ \\
\hline$\beta_{4}$ & 40.6 & $45.1 \pm 0.3$ \\
\hline$\delta_{2}$ & -7.4 & $-2.7 \pm 0.3$ \\
\hline
\end{tabular}

Table 2. Comparison between theoretical and experimentally measured Mach reflection shock and shear layer angles.

has the strongest signal and dominates the entire spectrum. Certain portions of the spectrum are also magnified in order to show the presence of $\mathrm{N}_{2}^{+}$and NO. Therefore, the identified emitters with temperaturefitting potential in this wavelength regime are $\mathrm{NO}, \mathrm{OH}$ and $\mathrm{N}_{2}^{+}$. Based on these results, the decision was made to concentrate efforts upon the ultraviolet portion of the spectrum. Additional measurements also detected no noticeable signatures in the visible region.

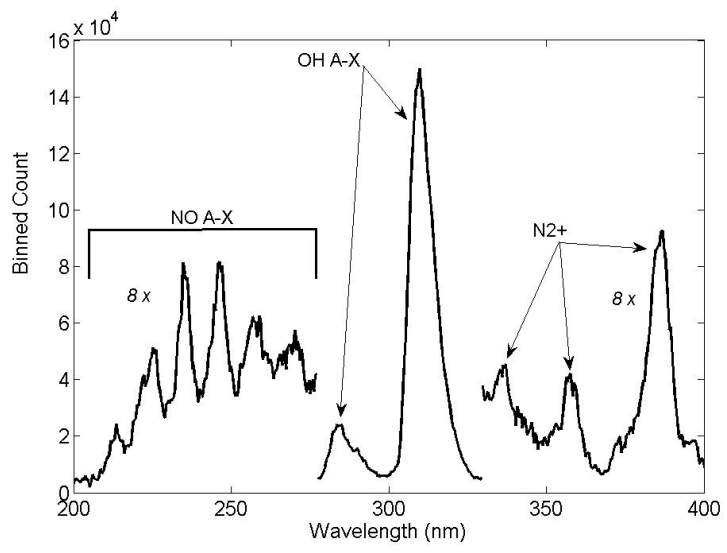

Figure 3. Spectrum obtained $10 \mathrm{~mm}$ downstream of the Mach stem with a 50 micron slit and 50 microsecond exposure time with certain portions of the spectrum magnified.

\section{D. $\mathrm{OH}$ results}

As a preliminary means of determining the feasibility of applying fine-scale resolution emission spectroscopy to this flow field, initial measurements were conducted using the emission spectra of $\mathrm{OH}$. These data collection locations with respect to the Mach reflection are shown in Figure 2 a). The $\mathrm{OH}$ A-X band was deliberately chosen for initial temperature-fitting as its calibration setup was easier to implement than the NO A-X band as a traditional blackbody radiation source could be used, which has an analytical solution. Additionally, $\mathrm{OH}$ is already present in the flow field due to moisture contamination in the test gas. Fine-scale spectra were collected and temperature fits constructed for the $A^{2} \sum^{+} \rightarrow X^{2} \prod$ electronic transition in the 307-320 region. An example of an emission spectrum for the $\mathrm{OH}$ A-X band obtained $4 \mathrm{~mm}$ downstream of the Mach stem is shown in Figure 4. Simulated and experimentally observed spectra are compared. At each axial location, LIFBASE $^{48}$ was used to generate simulated spectra across a broad temperature range at coarse temperature increments of $100 \mathrm{~K}$ using Lorentzian profiles and a resolution of $0.14 \mathrm{~nm}$. Wavelengths were interpolated so that the LIFBASE data points matched up with the experimental points and at each temperature increment a scaling factor was computed which reduces the residual, a value defined to be the difference between experimental and predicted intensity peaks. The smallest residual of the entire temperature range provided 
the fit temperature. Once the coarse temperature was determined, further optimization was performed such that the final temperature increment was $20 \mathrm{~K}$. As shown by Figure 4, the results indicate that the intensities across the entire spectrum match up very well and that reasonable temperature fits have been achieved. Spectra collected at $1 \mathrm{~mm}$ upstream of the Mach stem yielded no signal, confirming the spatial precision of the imaging location technique and lack of freestream dissociation in the facility.

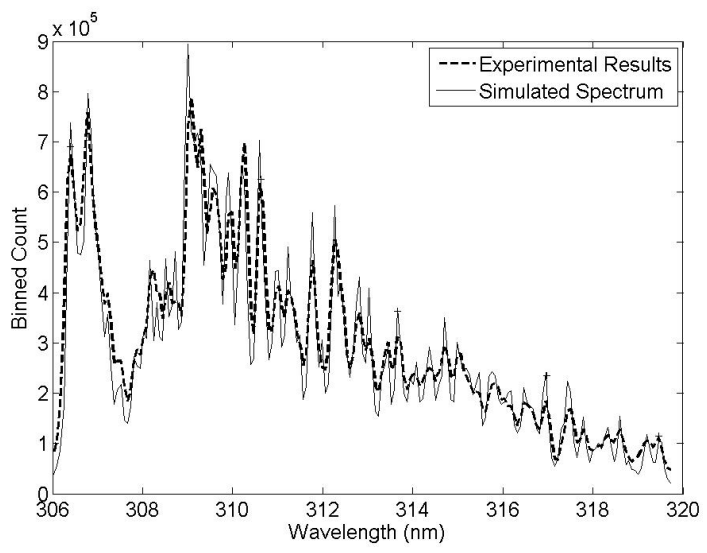

Figure 4. Simulated LIFBASE and experimental OH spectra obtained $4 \mathrm{~mm}$ downstream of the Mach stem for a vibrational temperature of $4140 \mathrm{~K}$.

\section{E. NO results}

Experiments were undertaken in order to investigate the NO $\gamma$ band. Interrogation of the $\gamma$ band permits comparison with numerical results as the thermochemical model described above does not include $\mathrm{OH}$ as a species. Figure 5 is an example of three NO spectra at three different streamwise locations of 2,3 and $4 \mathrm{~mm}$ downstream from the Mach stem. The graph indicates that the signal strength markedly attenuates with increasing axial distance and suggests that the experimental spatial precision is adequate.

Spectral fitting to the NO data using LIFBASE yielded very high temperatures, possibly due to the LIFBASE rotational and vibrational quantum number limits of 80 and 5 respectively. Calculations across an appropriate temperature range revealed that non-negligable Boltzmann rotational and vibrational populations exist for NO above these limits. Hence, an in-house algorithm developed by Glumac with user-defined maximum limits was used to generate simulated spectra. Vibrational and rotational energies are determined to the third and second-order respectively via Dunham-type potential expansions. For consistency, the spectroscopic constants used in these expansions are taken as those from LIFBASE. ${ }^{49}$ For the doublet ground and upper state energies, the analytical expressions of $\operatorname{Kovacs}^{50}$ were used and the dependence of the spin-orbit parameter, $A$, upon the vibrational quantum number is taken from Amiot. ${ }^{51}$ Line strengths were calculated via multiplication of the Franck-Condon factors and the Hönl-London factors. The Hönl-London factors for the doublet transitions were determined following the expressions of Kovacs ${ }^{50}$ and the Franck-Condon factors were taken from Ory et al. ${ }^{52}$ Conversion from vacuum to air wavelengths is achieved via the Edlen formula. The LIFBASE simulation formulates emission intensity as a function of molecule lifetime, emission co-efficients and predissociation rates. LIFBASE calculates the emission co-efficients by incorporating the Hönl-London factors and the transition probabilities that are calculated using the Rydberg-Klein-Ress (RKR) method. The predissociation phenomena has been observed for the NO $\gamma$ band and has been experimentally ${ }^{53}$ theoretically ${ }^{54}$ and numerically ${ }^{55}$ shown to be dependent upon both vibrational and rotational quantum numbers. Vibrational levels of $\mathrm{v}^{\prime}=4$ and 5 can predissociate and for $\mathrm{v}^{\prime}=3$, rotational levels above $\mathrm{N}^{\prime}=25$ can predissociate. ${ }^{53}$ However, for the most part transitions from these levels result in energies outside the current wavelength regime. As identified by Figure 6, the dominant vibrational transitions which weigh 


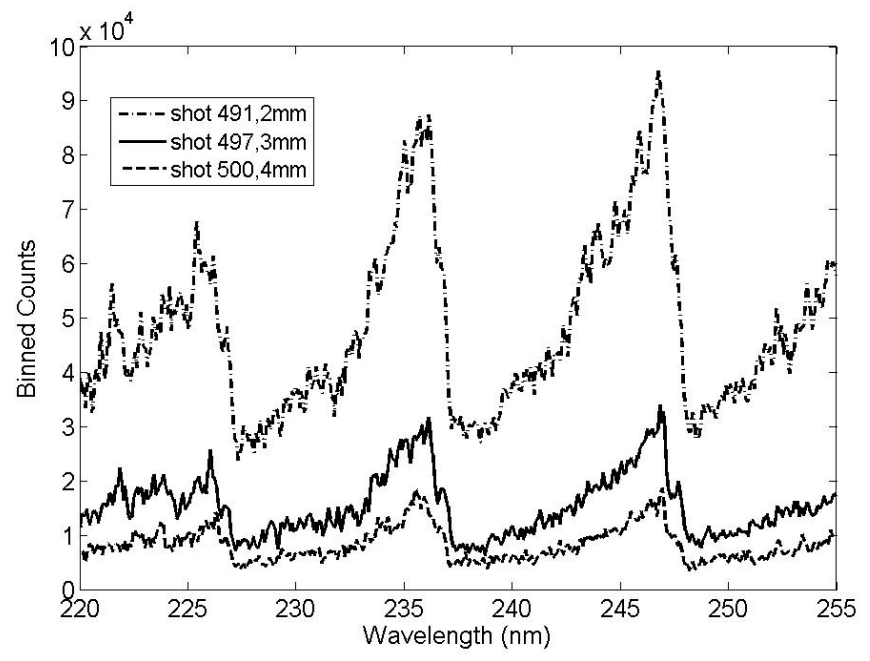

Figure 5. NO $\gamma$ band spectra obtained at three different locations (2,3 and $4 \mathrm{~mm})$ downstream of the Mach stem.

heavily upon the temperature-fitting procedure will not predissociate as explained above. Furthermore, LIFBASE simulations for 220-255 nm show no difference between spectra with and without predissociation broadening. Therefore, predissociation is not considered to be a phenomena which needs to be considered for the wavelength range of interest.

Figure 6 shows a comparison between LIFBASE and an in-house simulation for a temperature of $6200 \mathrm{~K}$ at the LIFBASE limits. The major vibrational band transitions are also indicated. The spectra are normalized by the maximum intensity in each case, which corresponds to the $(0,1)$ peak value. The agreement between the two simulations is very good and within the experimental spectral resolution. Figure 7 shows a comparison between the two custom simulations, one with the LIFBASE limits and the other with the extended limits of of $\nu_{\max }=20$ and $J_{\max }=250$. The extended vibrational limits (and hence larger $\Delta \mathrm{v}$ values) will not influence the simulated spectrum, however the modelling of extra rotational levels will alter the spectrum within the $220-255 \mathrm{~nm}$ range. The change in line strengths due to the additional levels justifies the decision to simulate higher rotational and vibrational levels.

Fine-scale spectra were collected and temperature fits constructed for the NO $A^{2} \sum^{+} \rightarrow X^{2} \prod$ electronic transition in the 220-255 nm region. The temperature fitting procedure was the same as described for the $\mathrm{OH}$ A-X transition. NO spectra were collected at 1, 2, 3 and $4 \mathrm{~mm}$ downstream of the Mach stem. Further downstream spectral acquisition of the relaxation region was restricted by the influence of converging shear layers bounding the measurement region, as shown in Figure 2 a). A sample spectrum, collected at $2 \mathrm{~mm}$ behind the Mach stem, is shown in Figure 8 along with the simulated comparison. The vibrational temperature in this case was determined to be $6900 \mathrm{~K}$. As exhibited for all imaging locations, the NO spectra are somewhat noisy and the visual fit fidelity is not as pronounced as in the $\mathrm{OH}$ spectra. However, the computational algorithm uses the line intensities as the optimizing parameter and as shown in Figure 8, the major vibrational band transition intensities are all very accurately matched.

The potential of contaminant emission within the 220-255 nm range was further investigated. Previous spectroscopic measurements at the TCM2 hypersonic facility have highlighted the importance of reducing test gas contamination. Pilverdier et al. noticed that a mylar secondary diaphragm displayed significantly less emissive intensity than one made from copper. ${ }^{24}$ The HET facility already operates with a mylar diaphragm. Ramjuan et al. also emphasized the importance of cleaning the facility. ${ }^{26}$ As a result, before each shot both the driven and accelerator section were cleaned. No differences in the nitric oxide spectra were observed. Dry air was also used as the test gas in order to see if the trace quantities of OH were contami- 


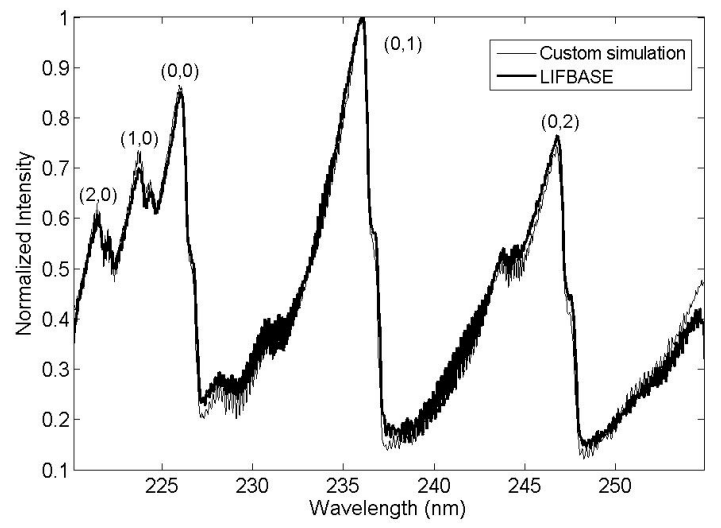

Figure 6. Comparison between the results of the in-house developed simulated spectrum and LIFBASE at a temperature of $6200 \mathrm{~K} . \nu_{\max }=5$ and $J_{\max }=80$.

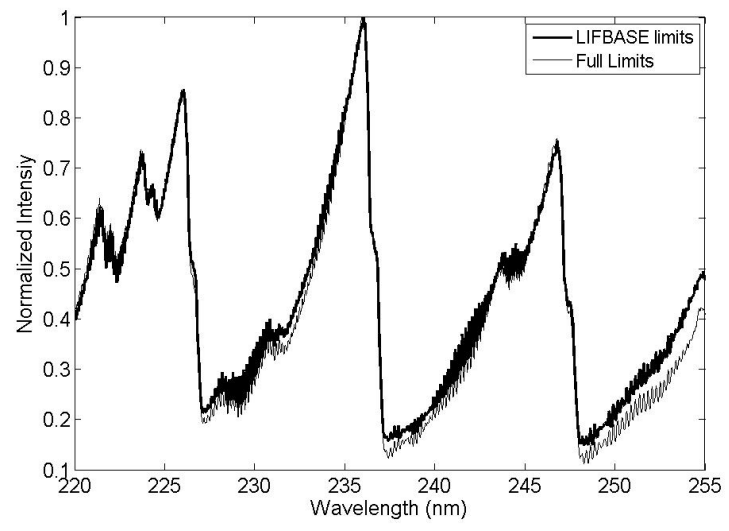

Figure 7. Comparison between the results of two custom simulated spectrums, one with LIFBASE limits and the other with the extended limits of $\nu_{\max }=20$ and $J_{\max }=\mathbf{2 5 0}$.

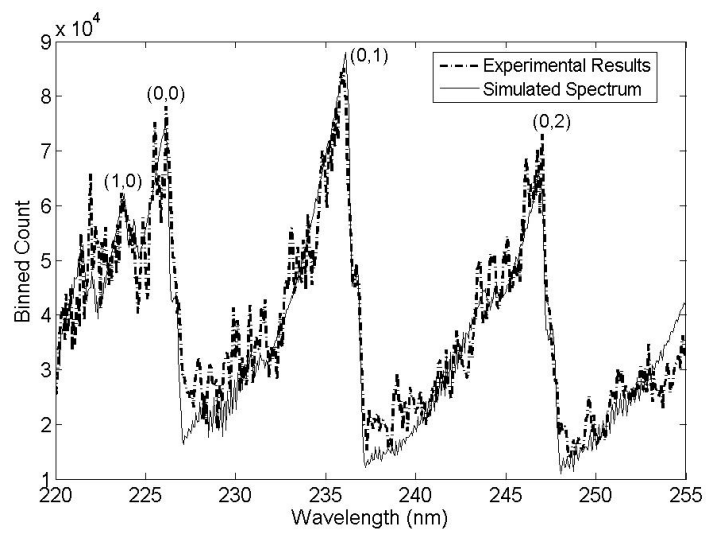

Figure 8. Simulated and experimental NO spectra obtained $2 \mathrm{~mm}$ downstream of the Mach stem for a vibrational temperature of $6900 \mathrm{~K}$. 
nating the results. The test location $2 \mathrm{~mm}$ behind the Mach stem was used to determine the influence of the moisture within the air. At this location five room air shots and six dry air shots were conducted and the temperatures determined. The standard deviation of these determined temperatures was $320 \mathrm{~K}$ and $140 \mathrm{~K}$ for the room air and dry air runs respectively. Despite the improvement in shot-to-shot repeatability with dry air as the test gas, no marked change in the experimental spectra was observed. The $\mathrm{O}_{2}$ Schumann-Runge band system has emission within the investigated spectral regime and is a common interference for UV NO air emission spectroscopic studies. ${ }^{15}$ As shown in Figure 8, possible $\mathrm{O}_{2}$ radiation is considerably less intense than the NO $\gamma$ signal and hence it is proposed that this interference is not significant enough to seriously hamper the temperature-fitting procedure.

Experimentally measured NO vibrational temperatures are shown in Figure 9. Data are obtained from three experiments each at 1, 3 and $4 \mathrm{~mm}$ and six experiments at the $2 \mathrm{~mm}$ location. The repeatability of the measurements is good. The temperature profile calculated using the detailed thermochemical model detailed above is also shown. Very good agreement is achieved between calculated and experimentally measured vibrational temperatures across the relaxation region. The largest discrepancy is observed at the $2 \mathrm{~mm}$ location, while the temperature measurements at the other three locations are all within experimental error. Table 3 compares the ensemble average of the temperature measurement at each location versus the numerical result. The results from the $\mathrm{OH}$ temperature fits described above are also presented on the same graph. The $\mathrm{OH}$ vibrational temperatures are considerably lower than those of $\mathrm{NO}$ and the temperature gradient throughout the relaxation region is much less pronounced and accompanied by a faster relaxation rate. $\mathrm{OH}$ error bars are not presented as no numerical simulation was conducted and hence no temperature gradient was available. The significant disparity between the experimentally measured $\mathrm{OH}$ and $\mathrm{NO}$ vibrational temperatures is indicative of the non-equilibrium flow field. At this stage, with no current $\mathrm{OH}$ thermochemical model available or further in-depth research, it is difficult to provide a physical explanation for this disparity. However, the experimentally measured $\mathrm{OH}$ vibrational temperatures within a canonical flow field provide comparative data for any $\mathrm{OH}$ thermochemical model.

The temperature measurement sources of error arise from shot-to-shot variation in MR location, the

\begin{tabular}{|l|l|l|}
\hline $\begin{array}{l}\text { Distance } \\
(\mathrm{mm})\end{array}$ & $\begin{array}{l}\text { Numerical } \\
(\mathrm{K})\end{array}$ & $\begin{array}{l}\text { Experimental } \\
(\mathrm{K})\end{array}$ \\
\hline 1 & 7143 & 7067 \\
\hline 2 & 6356 & 6917 \\
\hline 3 & 5971 & 6060 \\
\hline 4 & 5721 & 6007 \\
\hline
\end{tabular}

Table 3. Comparison between averaged experimental values and numerical results at different locations behind the Mach stem.

spatial precision of the imaging location, test condition variability, and the temperature increment selection of the fit to simulated spectra. The first two sources are dependent upon the relaxation region temperature gradient, which was determined from numerical calculations. As described in Section IV B, the uncertainties in the imaging location and MR movement are $0.25 \mathrm{~mm}$ and $0.5 \mathrm{~mm}$ respectively. The freestream static temperature was used as the parameter to gauge test condition variability. For the purpose of the uncertainty analysis, the freestream static temperature is taken to be only dependent upon the HET initial pressures.

\section{Conclusions}

Emission spectroscopy measurements of nitric oxide and hydroxl radical vibrational temperatures behind a strong normal shock in a hypervelocity flow field are presented. The stationary normal shock is created via a Mach reflection using an opposing wedge model in a high enthalpy Mach 7.42 freestream in an expansion tube. The inflow conditions for the normal shock have been experimentally defined by pressure, velocity and 


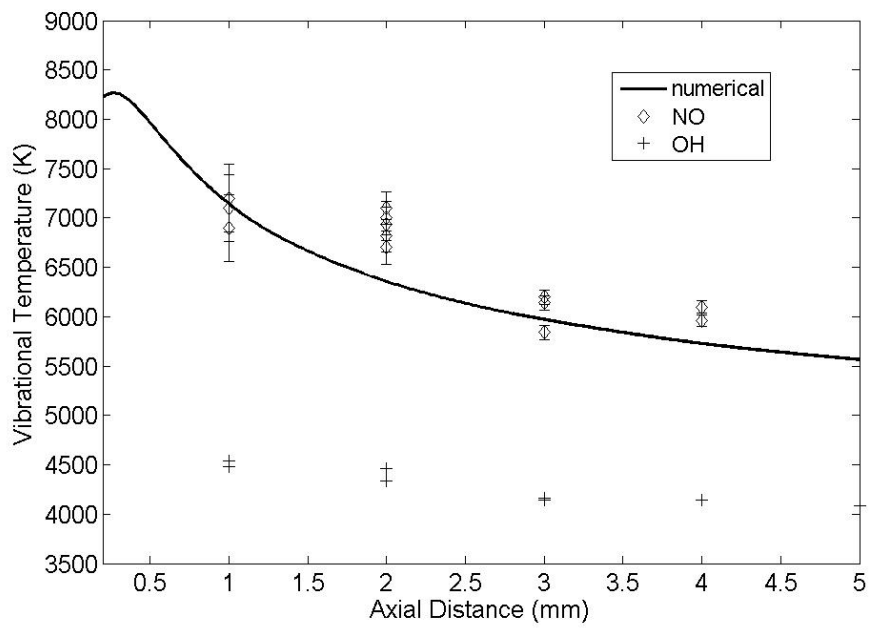

Figure 9. Experimentally measured $\mathrm{NO}$ and $\mathrm{OH}$ vibrational temperatures in the post-shock relaxation region. Measurements are compared to calculations using a detailed thermochemical model.

shock angle measurements. Experimental spectral data were collected at selected locations, $1 \mathrm{~mm}$ apart, behind the Mach stem. OH temperature fits were conducted using simulated spectra generated by LIFBASE, whereas the NO fits required higher vibrational and rotational levels and the use of an in-house spectral simulation algorithm. A state-resolved thermochemical model was used to model the temperature profile upto $5 \mathrm{~mm}$ downstream of the Mach stem. Agreement between NO vibrational measurements and computations is seen to be excellent, validating both the experimental approach and the non-equilbrium thermochemical model.

\section{Acknowledgments}

This research was funded in part through AFOSR/MURI Grant FA9550-04-1-0425 with Dr. John Schmisseur as Technical Monitor.

\section{References}

${ }^{1}$ Wen, C. Y. and Hornung, H. G., "Non-equilibrium dissociating flow over sphere," Journal of Fluid Mechanics, Vol. 299, 1995, pp. 389-405.

${ }^{2}$ Candler, G. V., Mavriplis, D. J., and Trevino, L., "Current Status and Future Prospects for the Numerical Simulation of Hypersonic Flows," AIAA Paper 2009-153, Vol. 47th AIAA Aerospace Sciences Meeting, 2009.

${ }^{3}$ M.Sharma, Austin, J. M., and Glumac, N. G., "Effect of thermochemistry on Mach reflection in hypervelocity flow," AIAA Paper 2008-5066, 2008.

${ }^{4}$ Nerem, R. M., Carlson, L. A., and Hartsel, J. E., "Chemical Relaxation Phenomena behind Nomral Shock Waves in a Dissociated Freestream," AIAA Journal, Vol. 5, No. 5, 1966, pp. 910-916.

${ }^{5}$ Russo, A. L., "Spectrophotometric Measurements of the Vibrational Relaxation of CO in Shock-Wave and Nozzle Expansion-Flow Environments," Journal of Chemical Physics, Vol. 47, No. 12, 1967, pp. 5201-5210.

${ }^{6}$ Moser, L. K. and Hindelang, F. J., "Vibrational relaxation of NO behind shock waves," Experiments in Fluids, Vol. 7 , No. 1, 1989, pp. 67-69.

${ }^{7}$ Moser, L. K. and Hindelang, F. J., "Shock-tube Study of the Vibrational Relaxation of Nitric Oxide," AIP Conference Proceedings - n. 208, Vol. 17th International Symposium on Shock Waves and Shock Tubes, Bethlehem, PA, 1990 , pp. 531-536.

${ }^{8}$ Sharma, S. P. and Gillespie, W., "Nonequilibrium and Equilibrium Shock Front Radiation Measurements," Journal of Thermophysics and Heat Transfer, Vol. 5, No. 3, 1991, pp. 257-265. 
${ }^{9}$ Allen, R. A., Keck, J. C., and Camm, J. C., "Non-Equilibrium Radiation from Shock Heated Nitrogen and a Determination of the Recombination Rate," AVCO-Everett Research Laboratory, Vol. Report. 110, 1961.

${ }^{10}$ Allen, R. A., "Nonequilibrium Shock Front Rotational, Vibrational and Electronic Temperature Measurements," AVCOEverett Research Laboratory, Vol. Report. 1986, 1964.

${ }^{11}$ Allen, R. A., Keck, J. C., and Camm, J. C., "Radiation from Hot Nitrogen," AVCO-Everett Research Laboratory, Vol. Report. 102, 1961.

${ }^{12}$ Labracherie, L., Billiotte, M., and Houas, L., "Nonequilibrium Determination of Temperature Profiles by Emission Spectroscopy," Journal of Quantitative Spectroscopy and Radiative Transfer, Vol. 54, No. 3, 1995, pp. 573-579.

${ }^{13}$ Ramjuan, D., Dumitrescu, M., and Brun, R., "Kinetics of Free Radicals Behind Strong Shock Waves," Journal of Thermophysics and Heat Transfer, Vol. 13, No. 2, 1999, pp. 219-225.

${ }^{14}$ Rond, C., Boubert, P., Felio, J. M., and Chikhaoui, A., "Nonequilibrium radiation behind a strong shock wave in CO2-N2," Journal of Chemical Physics, Vol. 340, No. 1-3, 2007, pp. 93-104.

${ }^{15}$ Wurster, W. H., Treanor, C. E., and Williams, M. J., "Nonequilibrium UV Radiation and Kinetics Behind Shock Waves in Air," AIAA Paper 1989-1918, Vol. AIAA 20th Fluid Dynamics, Plasma Dynamics and Lasers Conference, Buffalo, NY, 1989.

${ }^{16}$ Wurster, W. H., Treanor, C. E., and Williams, M. J., "Kinetics of UV Production Behind Shock Waves in Air," AIAA Paper 1990-1666, Vol. AIAA 21st Plasma Dynamics and Lasers Conference, Seattle, WA, 1990.

${ }^{17}$ Babikian, D. S., Gopaul, N. K. J. M., and Park, C., "Measurement and Analysis of Nitric Oxide Radiation in an Arcjet Flow," Journal of Thermophysics and Heat Transfer, Vol. 8, No. 4, 1994, pp. 737-743.

${ }^{18}$ Park, C. S., Newfield, M. E., Fletcher, D. G., and Gökçen, T., "Spectroscopic Measurements of Shock-Layer Flows in an Arcjet Facility," Journal of Thermophysics and Heat Transfer, Vol. 13, No. 1, 1999, pp. 60-67.

${ }^{19}$ Park, C. S., Newfield, M. E., Fletcher, D. G., G"okçen, T., and Sharma, S. P., "Spectroscopic Emission Measurements Within the Blunt-Body Shock Layer in an Arcjet Flow," Journal of Thermophysics and Heat Transfer, Vol. 12, No. 2, 1998, pp. 190-197.

${ }^{20}$ Staack, D., Farouk, B., Gutsol, A. F., and Fridman, A. A., "Spectroscopic studies and rotational and vibrational temperature measurements of atmospheric pressure nomral glow plasma discharges in air," Plasma Sources Science and Technology, Vol. 15, No. 4, 2006, pp. 818-827.

${ }^{21}$ Holden, M. S. and Candler, G. V., "Experimental studies in the LENS shock tunnel and expansion tunnel to examine real-gas effects in hypervelocity flows," AIAA Paper,2004-0916, Vol. 42nd AIAA Aerospace Sciences Meeting and Exhibit,Reno,Nevada, 2004

${ }^{22}$ I.Nompelis, Candler, G. V., and Holden, M. S., "Effect of Vibrational Nonequilibrium on Hypersonic Double-Cone Experiments," AIAA Journal, Vol. 41, No. 11, 2003, pp. 2162-2169.

${ }^{23}$ C.Park, "Thermochemical Relaxation in Shock Tunnels," Journal of Thermophysics and Heat Transfer, Vol. 4, No. 4, 2006, pp. 690-698.

${ }^{24}$ Pilverdier, H., Brun, R., and Dumitrescu, M. P., "Emission and Raman spectroscopy measurements in hypersonic nitrogen flows," Journal of Thermophysics and Heat Transfer, Vol. 15, No. 4, 2001, pp. 484-490.

${ }^{25}$ Boubert, P., Chaiz, A., Chikhaoui, A., Robin, L., and Versich, P., "Aerodynamic calibration of TCM2 facility and study of a bow shock layer by emission and laser spectroscopy," Shock Waves, Vol. 11, No. 5, 2002, pp. 341-351.

${ }^{26}$ Ramjuan, D., Sasoh, A., Ohnishi, Y., and Takayama, K., "Time-resolved spectroscopy in the shock layer of a blunt body placed in an expansion tube flow," 24th International Congress on High-Speed Photography and Photonics, Vol. 4183, 2001.

${ }^{27}$ Dufrene, A., Sharma, M., and Austin, J. M., "Design and characterization of a hypervelocity expansion tube facility," Journal of Propulsion and Power, Vol. 23, No. 6, 2007, pp. 1185-1193.

${ }^{28}$ McGilvray, M., Austin, J. M., Sharma, M., Jacobs, P. A., and Morgan, R. G., "Diagnostic modelling of an expansion tube operating condition," Shock Waves, Vol. 19, No. 1, 2009, pp. 59-66.

${ }^{29}$ Massa, L. and Austin, J. M., "Spatial linear stability of a hypersonic shear layer with nonequilibrium thermochemistry," Physics of Fluids, Vol. 20, No. 8, 2008, pp. 84-104.

${ }^{30}$ Adamovich, I. and Rich, J., "Three Dimensional Nonperturbative Analytical Model of Vibrational Energy Transfer in Atom-Molecule Collisions," Journal of Chemical Physics, Vol. 109, No. 18, 1998, pp. 7711-7724.

${ }^{31}$ Macheret, S. and Adamovich, I., "Semiclassical Modelling of State Specific Dissociation Rates in Diatomic Gases," Journal of Chemical Physics, Vol. 113, No. 17, 2000, pp. 7351-7361.

32 Adamovich, I. V., "Three dimensional analytical model of vibrational energy transfer in molecule-molecule collisions," AIAA J., Vol. 39, 2001, pp. 10.

${ }^{33}$ Bose, D. and Candler, G. V., "Thermal Rate Constants of the $\mathrm{N}_{2}+\mathrm{O} \rightarrow \mathrm{NO}+\mathrm{N}$ Reaction Using $a b$ initio Potential Energy Surfaces," Journal of Chemical Physics, Vol. 10, No. 8, 1996, pp. 2825-2833.

${ }^{34}$ Bose, D. and Candler, G. V., "Thermal Rate Constants of the $\mathrm{O}_{2}+\mathrm{N} \rightarrow \mathrm{NO}+\mathrm{O}$ Reaction Based on ab initio Potential Energy Surfaces," Journal of Chemical Physics, Vol. 107, No. 16, 1997, pp. 6136-6145.

${ }^{35}$ Kolesnick, G. D. B. R. E., "Vibrational Relaxation of Oxygen. State to State Rate Constants," Chemical Physics Letters, Vol. 200, No. 4, 1992, pp. 382-386.

${ }^{36}$ Billing, G. D., "Rate Constants and Cross Sections for Vibrational Transitions in Atom-Diatom and Diatom-Diatom Collisions," Computer Physics Communications, Vol. 32, No. 1, 1984, pp. 45-62. 
${ }^{37}$ Millikan, R. C. and White, D. R., "Systematic of Vibrational Relaxation," Journal of Chemical Physics, Vol. 39, No. 12, 1963, pp. 3209-3213.

${ }^{38}$ Ormonder, S., "Vibrational Relaxation Theories and Measurements," Review of Modern Physics, Vol. 47, No. 1, 1975, pp. 193-258.

${ }^{39}$ Baulch, D., Drysdale, D., and Horne, D., "Evaluated Kinetic Data for High Temperature Reactions," CRC, Boca Raton, Vol. 2-3.

${ }^{40}$ Camac, M. and Vaughan, A., "O ${ }_{2}$ Dissociation Rates in $\mathrm{O}_{2}$-Ar Mixtures," Journal of Chemical Physics, Vol. 34, No. 2, 1961, pp. 460-470.

${ }^{41}$ Taylor, R. L., Camac, M., and Feinberg, R. M., "Measurements of Vibration-Vibration Coupling in Gas Mixtures," Avco Everett Reseach Lab Report, RR-250, 1966, pp. 43p.

${ }^{42}$ Skews, B. W., "Aspect ratio effects in wind tunnel studies of shock wave reflection transition," Shock Waves, Vol. 7 , 1997, pp. 373-383.

${ }^{43}$ Gimelschein, S., Markelov, G., and Ivanov, M., "Relaxation and rarefaction effects on shock wave reflections," AIAA Paper 98-2819, 1998.

${ }^{44}$ Grasso, F. and Paoli, R., "An Analytical Study of Mach Reflection in Nonequilibrium Steady Flows," Physics of Fluids, Vol. 11, No. 10, 1999, pp. 3150-3167.

${ }^{45}$ Burtschell, Y., Zeitoun, D. E., and Ben-Dor, G., "Steady shock wave reflections in thermochemical nonequilibrium flows," Shock Waves, Vol. 11, 2000, pp. 15-21.

${ }^{46}$ Grasso, F. and Paoli, R., "Simulations of Shock-Wave Reflections in Nonequilibrium Flows," Journal of Spacecraft and Rockets, Vol. 37, No. 2, 2000, pp. 224-234.

${ }^{47}$ McGilvray, M., Austin, J. M., Sharma, M., Jacobs, P. A., and Morgan, R. G., "Diagnostic modelling of an expansion tube operating condition for a hypersonic shear layer experiment," Proceedings of the 16th Australasian Fluid Mechanics Conference, 2007.

${ }^{48}$ Luque, J. and Crosley, D. R., "LIFBASE: Database and Spectral Simulation Program (Version 1.5)," SRI International Report MP 99-009, 1999.

${ }^{49}$ Luque, J. and Crosley, D. R., LIFBASE (version 1.9), 1999.

${ }^{50}$ Kovacs, I., Rotational Structure in the Spectra of Diatomic Molecules, 1969.

${ }^{51}$ Amiot, C., "The Infrared Emission Spectrum of NO: Analysis of the $\Delta \mathrm{v}=3$ Sequence up to v=22," Journal of Molecular Spectroscopy, Vol. 94, 1982, pp. 150-172.

${ }^{52}$ Ory, H. A., Gittleman, A. P., and Maddox, J. P., "Franck-Condon Factors for the NO Beta and Gamma Band Systems," Astrophysical Journal, Vol. 139, No. 1, 1963, pp. 346-356.

${ }^{53}$ Luque, J. and Crosley, D. R., "Radiative and predissociative rates for $\mathrm{NO} \mathrm{A}^{2} \sum^{+} \mathrm{v}^{\prime}=05$ and $\mathrm{D}^{2} \sum^{+} \mathrm{v}^{\prime}=0-3$, , Journal of Chemical Physics, Vol. 112, No. 21, 2000, pp. 9411-9416.

${ }^{54}$ Luque, J. and Crosley, D. R., "Transition probabilities and electronic transition moments of the $A^{2} \sum^{+} \rightarrow X^{2} \prod$ and $D^{2} \sum^{+} \rightarrow X^{2} \prod$ systems of nitric oxide," Journal of Chemical Physics, Vol. 111, No. 16, 1999, pp. 7405-7415.

${ }^{55}$ Velasco, A. M., Martin, I., Lavin, C., and Bustos, E., "Radiative lifetimes for the $\mathrm{A}^{2} \sum^{+}\left(\mathrm{v}^{\prime}=0-7\right)$ and $\mathrm{D}^{2} \sum^{+}\left(\mathrm{v}^{\prime}=0-5\right)$ Rydberg states of NO," Molecular Physics, Vol. 103, No. 10, 2005, pp. 1375-1385. 